\section{Psychological Medicine}

cambridge.org/psm

\section{Review Article}

Cite this article: Kothgassner OD, Goreis A, Robinson K, Huscsava MM, Schmahl C, Plener PL (2021). Efficacy of dialectical behavior therapy for adolescent self-harm and suicidal ideation: a systematic review and metaanalysis. Psychological Medicine 51, 1057-1067. https://doi.org/10.1017/S0033291721001355

Received: 23 October 2020

Revised: 22 March 2021

Accepted: 26 March 2021

First published online: 20 April 2021

\section{Keywords:}

Adolescence; borderline personality disorder; DBT-A; self-harm; self-injury; suicidal ideation

Author for correspondence:

Oswald D. Kothgassner,

E-mail: oswald.kothgassner@meduniwien.ac.at

\title{
Efficacy of dialectical behavior therapy for adolescent self-harm and suicidal ideation: a systematic review and meta-analysis
}

\section{Oswald D. Kothgassner ${ }^{1}$ (D), Andreas Goreis ${ }^{2,3}$ (D), Kealagh Robinson ${ }^{4}$ (D), Mercedes M. Huscsava ${ }^{1}$ (D), Christian Schmahl ${ }^{5}$ (D) and Paul L. Plener S $^{1,6}$ (D)}

${ }^{1}$ Department of Child and Adolescent Psychiatry, Medical University of Vienna, Vienna, Austria; ${ }^{2}$ Department of Clinical and Health Psychology, Faculty of Psychology, University of Vienna, Vienna, Austria; ${ }^{3}$ Outpatient Unit for Research, Teaching and Practice, Faculty of Psychology, University of Vienna, Vienna, Austria; ${ }^{4}$ School of Psychology, Te Herenga Waka - Victoria University of Wellington, Wellington, New Zealand; ${ }^{5}$ Department of Psychosomatic Medicine and Psychotherapy, Central Institute of Mental Health, Medical Faculty Mannheim, Heidelberg University, Mannheim, Germany and ${ }^{6}$ Department of Child and Adolescent Psychiatry and Psychotherapy, University of Ulm, Ulm, Germany
(C) The Author(s), 2021. Published by Cambridge University Press. This is an Open Access article, distributed under the terms of the Creative Commons Attribution licence (http://creativecommons.org/licenses/by/4.0/), which permits unrestricted re-use, distribution, and reproduction in any medium, provided the original work is properly cited.

\section{CAMBRIDGE UNIVERSITY PRESS}

\begin{abstract}
Background. Given the widespread nature and clinical consequences of self-harm and suicidal ideation among adolescents, establishing the efficacy of developmentally appropriate treatments that reduce both self-harm and suicidal ideation in the context of broader adolescent psychopathology is critical.

Methods. We conducted a systematic review and meta-analysis of the Dialectical Behaviour Therapy for Adolescents (DBT-A) literature on treating self-injury in adolescents (12-19 years). We searched for eligible trials and treatment evaluations published prior to July 2020 in MEDLINE/PubMed, Scopus, Google Scholar, EMBASE, and the Cochrane Library databases for clinical trials. Twenty-one studies were identified [five randomized-controlled trials (RCTs), three controlled clinical trials (CCTs), and 13 pre-post evaluations]. We extracted data for predefined primary (self-harm, suicidal ideation) and secondary outcomes (borderline personality symptoms; BPD) and calculated treatment effects for RCTs/CCTs and pre-post evaluations. This meta-analysis was pre-registered with OSF: osf.io/v83e7.

Results. Overall, the studies comprised 1673 adolescents. Compared to control groups, DBTA showed small to moderate effects for reducing self-harm $(g=-0.44 ; 95 \%$ CI -0.81 to $-0.07)$ and suicidal ideation $(g=-0.31,95 \%$ CI -0.52 to -0.09$)$. Pre-post evaluations suggested large effects for all outcomes (self-harm: $g=-0.98,95 \%$ CI -1.15 to -0.81 ; suicidal ideation: $g=-1.16,95 \% \mathrm{CI}-1.51$ to -0.80 ; BPD symptoms: $g=-0.97,95 \% \mathrm{CI}-1.31$ to $-0.63)$.

Conclusions. DBT-A appears to be a valuable treatment in reducing both adolescent selfharm and suicidal ideation. However, evidence that DBT-A reduces BPD symptoms was only found in pre-post evaluations.
\end{abstract}

Self-injury, suicidal ideation, and suicide attempts represent major mental health concerns for adolescents around the world. Suicide is the leading cause of death for female adolescents and the third highest cause of death for male adolescents in the western world (Collaboration GBoDP, 2016; Hawton, Saunders, \& O'Connor, 2012). In addition, meta-analyses estimate that $22.9 \%$ of adolescents have engaged in self-harm whereby they deliberately and directly damage their body tissue in the absence of suicidal ideation (Gillies et al., 2018). Although there is ongoing debate about the nature of the relationship between self-harm and suicidal thoughts and behaviors (Hamza, Stewart, \& Willoughby, 2012), the two are related (Gillies et al., 2018). Meta-analyses of longitudinal studies estimate that people who engage in selfharm have 4.27 greater odds of subsequently attempting suicide, and those who engage in deliberate self-harm - regardless of suicidal intent - have 1.51 greater odds of subsequent death by suicide (Ribeiro et al., 2016).

Adolescence represents a key developmental period for both self-harm and suicidality (Wyman, 2014). Although suicide is uncommon before the age of 15, the prevalence of suicide strongly increases from late adolescence to early adulthood (Bertolote \& Fleischmann, 2002). Self-harm also tends to begin during early adolescence around 13-15 years, with growing evidence to suggest that earlier onset of self-harm increases the risk of a more severe trajectory (Ammerman, Jacobucci, Kleiman, Uyeji, \& McCloskey, 2018; Groschwitz et al., 2015; Plener, Schumacher, Munz, \& Groschwitz, 2015). Self-harm and suicidal ideation typically present alongside other psychiatric disorders, such as affective and stress-associated disorders (Nock, Joiner, Gordon, Lloyd-Richardson, \& Prinstein, 2006), and among adults the most 
well-established link is with borderline personality disorder (BPD; Ferrara et al., 2012; Kaplan et al., 2016). Given the widespread nature and clinical consequences of adolescent self-injury, establishing developmentally appropriate treatments that reduce both self-harm and suicidal ideation in the context of broader adolescent psychopathology is critical.

One treatment which has received growing interest is Dialectical Behaviour Therapy for Adolescents (DBT-A). DBT was initially developed to treat women diagnosed with BPD at high-risk for suicide (e.g. Chapman, 2006; Linehan, Heard, \& Armstrong, 1993) and is widely recommended as an established therapeutic approach for people with BPD, particularly when reducing self-injury is a priority (APA, 2006; National Collaborating Center for Mental Health, 2009). Subsequently, a dialectical behavioral approach was adapted for adolescents, which prioritizes self-harm and suicidal ideation as the primary targets for therapeutic intervention (Miller, Rathus, \& Linehan, 2017; Rathus \& Miller, 2002). DBT-A is a manualized treatment approach intended for outpatient settings comprised of weekly individual therapy with concurrent participation in a skills-group and which includes parental participation. In particular, DBT-A focuses on developing mindfulness, distress tolerance, interpersonal effectiveness, and emotion regulation behavioral skills as the main therapeutic tools for overcoming pervasive emotion dysregulation and suicidal ideation (Miller et al., 2017; Rathus \& Miller, 2015).

To date, two reviews evaluating the efficacy of psychosocial treatments for reducing adolescent self-harm and suicidal ideation have highlighted DBT-A as a promising treatment (Glenn, Franklin, \& Nock, 2015; Kothgassner, Robinson, Goreis, Ougrin, \& Plener, 2020). However, previous meta-analysis focused exclusively on a small number $(k=3)$ of randomized controlled trials (RCTs; Kothgassner et al., 2020), and the systematic review of controlled clinical trials (CCTs, trials including a control group, but which lack randomization) and pre-post evaluations only included studies published prior to July 2013 ( $k=5$, no RCTs; Glenn et al., 2015). Given both the clinical importance of responding effectively to adolescent self-harm and suicidal ideation, and the limited number of DBT-A RCTs highlighted in previous reviews, we decided to include all studies across different stages of clinical evaluation in order to provide the most comprehensive synthesis of the current evidence. In addition, although DBT has shown success in treating BPD symptoms in adults, the efficacy of DBT-A for treating BPD symptoms among adolescents who self-injure remains to be evaluated (Cristea et al., 2017). Thus, in this review, we include RCTs, CCTs, and pre-post evaluation studies to evaluate the efficacy of DBT-A for reducing self-harm, suicidal ideation and BPD symptoms among adolescents, and conduct subgroup analyses to compare the results for RCTs with those of less rigorous studies. The greater heterogeneity in studies also allows us to assess whether characteristics of the study (e.g. participant age, treatment duration) moderate the meta-analytic effect of DBT-A on outcomes, in order to better understand the parameters under which DBT-A is most successful.

\section{Method \\ Search strategy and inclusion criteria}

We conducted a search of MEDLINE/PubMed, Scopus, Google Scholar, EMBASE, and the Cochrane Library databases for clinical trials for studies published from the beginning of database records until 31 July 2020 using the keywords 'Dialectical Behaviour Therapy' OR 'DBT-A' and combinations of the keywords 'Self-harm' OR 'Self-Injury', 'Suicidal Ideation', OR 'Suicide' with an age limitation. Studies were included in the meta-analysis if they reported an RCT or CCT comparing DBT-A with a control intervention or a pre-post evaluation of DBT-A and reported outcomes for self-harm and/or suicidal ideation in adolescents aged 12-19 who had engaged in selfinjury at least once. We also excluded studies focusing solely on pharmacological treatments. No limitations on language or publication status were invoked, and no other inclusion or exclusion criteria were applied.

We analyzed the frequency of self-harm episodes and suicidal ideation as primary outcome measures, with BPD symptoms as a secondary outcome measure. The title, abstract, and main text of each study were examined, with the exclusion of documents occurring at each stage. The initial search generated 932 results. Title and abstracts were screened for eligibility and full-text papers were obtained where necessary to evaluate inclusion. After screening, 21 studies - all peer-reviewed journal articles in English were identified and included in our meta-analysis.

\section{Data extraction and analysis}

Data from included studies were entered into a spreadsheet independently by two authors (ODK and KR). A third author (AG) reviewed and discussed differences until consensus was reached. We coded the sample and intervention characteristics of each study included in the meta-analysis. For analyses of the efficacy of DBT-A in RCTs and CCTs, the primary outcome was the standardized mean difference (Hedges' $g$ ) between the DBT-A and control interventions on self-harm and suicidal ideation measured post-intervention. The secondary outcome was the standardized mean difference (Hedges' $g$ ) for BPD symptoms in the DBT-A and control interventions measured post-intervention. For analyses regarding pre-post treatment effects, we computed the standardized mean difference (Hedges' $g$ ) based on means and standard deviations (Dunlap et al., 1996) before and after DBT-A intervention using the formula $g=\left(M_{\text {post }}-M_{\text {pre }}\right) /$ $\mathrm{SD}_{\text {pooled }}$, where $M_{\text {post }}$ is the mean of the measure after the intervention and $M_{\text {pre }}$ the mean before the intervention, with $\mathrm{SD}_{\text {pooled }}$ as the standard deviation for both measurements, defined as $\mathrm{SD}_{\text {pooled }}=\mathrm{SQRT}\left(\mathrm{SD}_{\text {pre }}^{2}+\mathrm{SD}_{\text {post }}^{2}\right) / 2$ (Lakens, 2013).

Means, standard deviations, and sample sizes were retrieved and inserted into a spreadsheet. If means or standard deviations were not reported in studies or Supplemental materials, conversions via Revman Calculator (Cochrane Collaboration, 2014) or formulas (Card, 2012) were conducted. If self-harm episodes were reported as proportions or odds ratios, they were transformed to Hedges' $g$ via the formula provided in Lipsey and Wilson (2001). Effect size calculations and meta-analyses were conducted with the metafor package for R (Viechtbauer, 2010). Following established conventions, an effect size of 0.20 was considered a small effect size, 0.50 a medium effect, and 0.80 a large effect size (Cohen, 1988). Random-effects models were applied to estimate aggregated effect sizes. Heterogeneity across study outcomes was reported with $I^{2}$ values, where $25 \%$ indicates low heterogeneity, $50 \%$ moderate, and $75 \%$ high heterogeneity (Higgins, Thompson, Deeks, \& Altman, 2003). Moderator analyses (meta-regression) were conducted to test whether treatment duration, gender composition, and participant mean age moderated the effect of the DBT-A on each outcome. Egger's regressions 


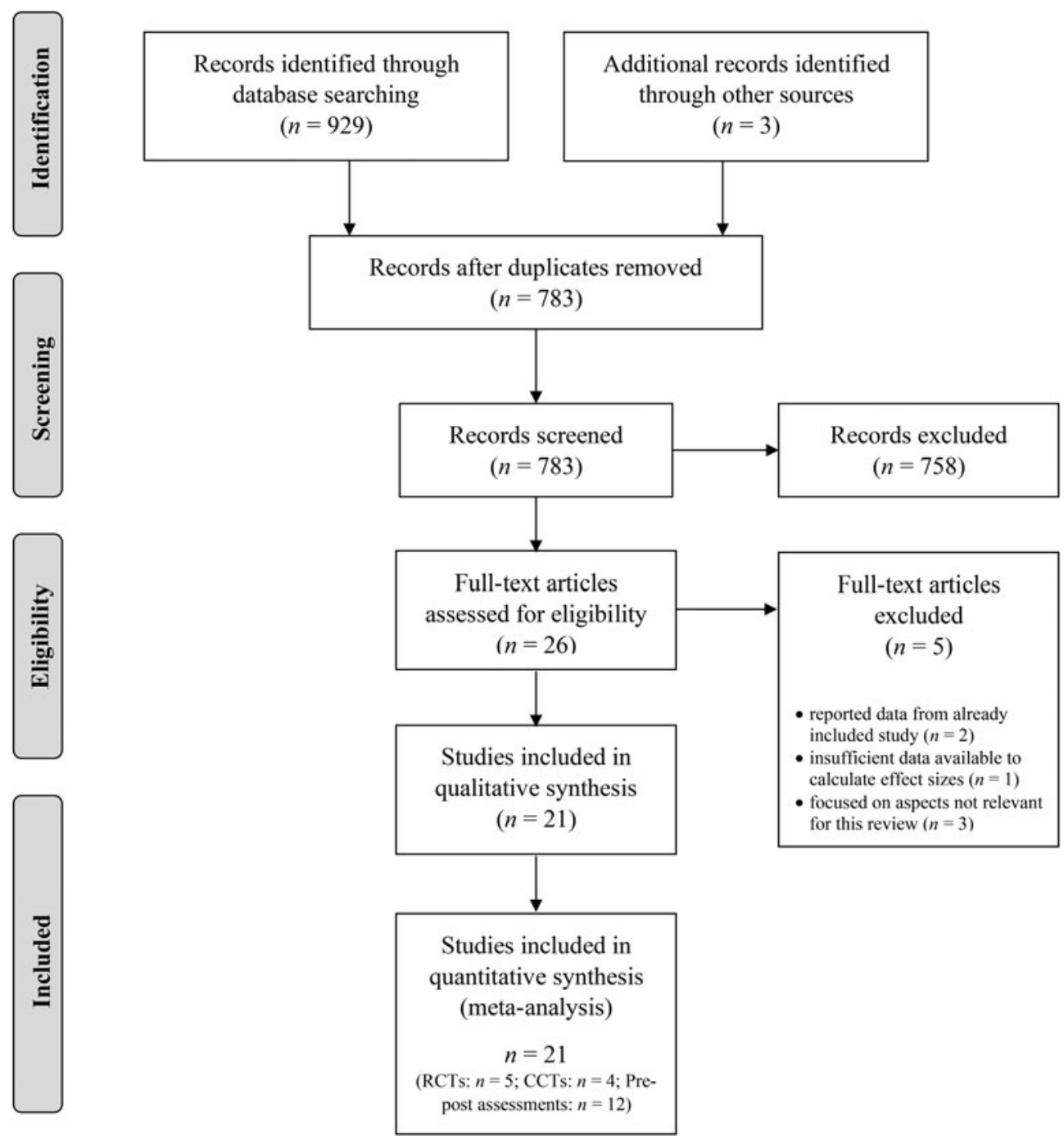

Fig. 1. PRISMA flowchart showing the screening, exclusion, and inclusion criteria.

were conducted to estimate publication bias (Sterne \& Egger, 2005), with adjusted effect sizes calculated using trim-and-fill analyses and, based on funnel plot asymmetry, numbers of imputed missing studies (Duval \& Tweedie, 2000). All data and analysis code are available on the Open Science Framework (doi:10.17605/OSF.IO/YZXPJ).

\section{Risk of bias assessment}

Risk of bias for each study was assessed using predefined criteria based on the Agency for Healthcare Research and Quality method guide (see Supplement 1; Viswanathan et al., 2018). Each study was assessed in regard to randomization, selection and attrition bias, confounding bias, measurement bias, and statistical problems and received a rating of low, moderate or high risk of bias. Low risk of bias indicates that the study was judged to be valid, moderate risk indicates concerns that probably do not invalidate the study's results, and high risk of bias indicates significant concerns that likely invalidate the study's results. Two investigators (KR and ODK) independently assessed the studies and differences were reviewed until consensus was reached.

\section{Results}

In total, 21 studies were identified (see Fig. 1 for the PRISMA flow diagram). Five studies were RCTs, three studies were CCTs, and 13 were pre-post evaluation studies (see Table 1 for an overview of study characteristics). The study by Rathus and Miller (2002) - originally included as a CCT - was included as a prepost evaluation study, given that data for the control intervention was unavailable. In total, the 21 studies comprised 1673 adolescents. Overall, 1063 participants received DBT-A interventions, and 610 received control interventions. A sufficient number of studies $(k>1$; Pigott, 2012) were identified to calculate aggregate effect sizes for self-harm and suicidal ideation outcomes in RCTs, CCTs, and pre-post evaluations. However, only one controlled study reported BPD symptoms as an outcome, and so this outcome was solely assessed among pre-post evaluations. Across studies, participants tended to be female $(M=82 \%)$ and 15.4 years old (s.D. $=1.3$ ). An average of $7 \%$ of participants droppedout across studies (range: $0-40 \%$ ) and $63 \%$ received concurrent psychopharmacological intervention over the course of the intervention $(k=11$ studies did not provide sufficient data about medication). 


\begin{tabular}{|c|c|c|c|c|c|c|c|c|c|c|c|}
\hline Study Year & $\begin{array}{l}\text { Study } \\
\text { design }\end{array}$ & $\begin{array}{l}\text { Sample } \\
\text { size }\end{array}$ & Country & $\begin{array}{l}\text { Control } \\
\text { intervention }\end{array}$ & $\begin{array}{c}\% \\
\text { Female }\end{array}$ & $\begin{array}{l}\text { Age range } \\
\text { (mean) }\end{array}$ & $\begin{array}{l}\text { Treatment } \\
\text { duration in } \\
\text { months }\end{array}$ & Setting & Eligibility criteria & $\begin{array}{l}\text { Outcome } \\
\text { (measures) }\end{array}$ & $\begin{array}{c}\% \\
\text { Drop-out }\end{array}$ \\
\hline $\begin{array}{l}\text { Apsche, Bass, and Siv } \\
(2006)\end{array}$ & $\mathrm{RCT}$ & 20 & USA & $\begin{array}{l}\text { Mode deactivation } \\
\text { therapy }\end{array}$ & $0 \%$ & $\begin{array}{l}\text { 15-18 (DBT-A: } \\
\text { 15.9, Control: } \\
16.1 \text { ) }\end{array}$ & 12 & $\begin{array}{l}\text { Residential } \\
\text { treatment }\end{array}$ & Not stated & SI (SIQ) & $0 \%$ \\
\hline $\begin{array}{l}\text { Berk, Starace, Black, } \\
\text { and Avina }(2020)\end{array}$ & Pre-post & 22 & USA & - & $92 \%$ & $12-17(15.2)$ & 6 & Outpatient & $\begin{array}{l}\text { Recent history of suicidal and/or } \\
\text { self-injury behaviors }\end{array}$ & $\begin{array}{l}\text { SH (past 6-month } \\
\text { frequency), SI (SIQ/ } \\
\text { SIQ-Jr), BPD } \\
\text { (SCID-II) }\end{array}$ & $8 \%$ \\
\hline Buerger et al. (2019) & Pre-post & 72 & Germany & - & $92 \%$ & $12-17(15.7)$ & 6.25 & Outpatient & $\begin{array}{l}3+B P D \text { criteria; fluency in } \\
\text { German }\end{array}$ & $\begin{array}{l}\text { SH and SI (SITBI-G), } \\
\text { BPD (LPI) }\end{array}$ & $18 \%$ \\
\hline $\begin{array}{l}\text { Courtney and } \\
\text { Flament (2015) }\end{array}$ & Pre-post & 42 & Canada & - & $93 \%$ & $\begin{array}{l}\text { Adolescents } 15 \\
\text { and above } \\
\text { (16.5) }\end{array}$ & 3 & Outpatient & $\begin{array}{l}\text { SIQ score }>30 \text {, or self-injury in the } \\
\text { past } 4 \text { months }\end{array}$ & $\begin{array}{l}\text { SH (chart review) } \\
\text { BPD (LPI) }\end{array}$ & $51 \%$ \\
\hline $\begin{array}{l}\text { Fischer and Peterson } \\
\text { (2015) }\end{array}$ & Pre-post & 7 & USA & - & $100 \%$ & $14-17(16.2)$ & 6 & Outpatient & $\begin{array}{l}\text { Binge eating within past } 4 \text { weeks; } \\
1+\text { suicide attempt or episode of } \\
\text { self-injury within past year; } \\
\text { height and weight within or } \\
\text { above typical limits for age and } \\
\text { stage of development }\end{array}$ & $\begin{array}{l}\text { SH (DSHI past } \\
\text { month) }\end{array}$ & $30 \%$ \\
\hline $\begin{array}{l}\text { Fleischhaker et al. } \\
\text { (2011) }\end{array}$ & Pre-post & 10 & Germany & - & $100 \%$ & $\begin{array}{l}13-19 \text { (not } \\
\text { stated) }\end{array}$ & 6 & Outpatient & $\begin{array}{l}\text { SH and/or suicidal behavior } \\
\text { within past } 16 \text { weeks; BPD } \\
\text { diagnosis or } 3+\text { BPD criteria }\end{array}$ & $\mathrm{SH}$ (LPC) & $25 \%$ \\
\hline $\begin{array}{l}\text { Geddes, Dziurawiec, } \\
\text { and Lee (2013) }\end{array}$ & Pre-post & 6 & Australia & - & $100 \%$ & $\begin{array}{l}14 \text { years } 6 \\
\text { month-15 } \\
\text { years } 1 \text { month } \\
(15.1)\end{array}$ & 6.5 & Outpatient & $\begin{array}{l}\text { Average cognitive ability and } \\
\text { established reading level; } \\
\text { self-injury and/or suicidal } \\
\text { ideation within past } 12 \text { months; } 3 \\
\text { + BPD criteria }\end{array}$ & $\begin{array}{l}\text { SH and SI (in-house } \\
\text { questionnaire) }\end{array}$ & $33 \%$ \\
\hline $\begin{array}{l}\text { Gillespie, Joyce, } \\
\text { Flynn, and Corcoran } \\
\text { (2019) }\end{array}$ & Pre-post & 84 & Ireland & - & $85 \%$ & 13-18 (15.7) & 4 & Outpatient & $\begin{array}{l}\text { Demonstration of emotional and } \\
\text { behavioral dysregulation; } \\
\text { persistent self-injury with } 1+ \\
\text { episodes of self-injury or suicidal } \\
\text { acts within the past } 16 \text { weeks or } \\
\text { chronic suicidal ideation; } \\
\text { expressed commitment by both } \\
\text { adolescent and parent/guardian }\end{array}$ & $\begin{array}{l}\text { SH (review of diary } \\
\text { cards), SI (in-house } \\
\text { questionnaire), } \\
\text { BPD (BSL-23) }\end{array}$ & $16 \%$ \\
\hline $\begin{array}{l}\text { Goldstein, Axelson, } \\
\text { Birmaher, and Brent } \\
\text { (2007) }\end{array}$ & Pre-post & 10 & USA & - & $80 \%$ & $14-18(15.8)$ & 6 & Outpatient & $\begin{array}{l}\text { Bipolar diagnosis with an acute } \\
\text { manic, mixed, or depressive } \\
\text { episode within past } 3 \text { months; } \\
\text { engaged in a pharmacotherapy } \\
\text { regimen; } 1+\text { parent/guardian } \\
\text { willing to participate in family } \\
\text { sessions }\end{array}$ & $\begin{array}{l}\text { SH (K-SADS), SI } \\
\text { (MSSI) }\end{array}$ & $10 \%$ \\
\hline Goldstein et al. (2015) & RCT & 20 & USA & Treatment-as-usual & $75 \%$ & $\begin{array}{l}\text { 12-18 (DBT: } \\
\text { 15.8, Control: } \\
16.8 \text { ) }\end{array}$ & 12 & Outpatient & $\begin{array}{l}\text { Bipolar disorder diagnosis; an } \\
\text { acute manic, mixed, or } \\
\text { depressive episode within past } 3 \\
\text { months; willingness to engage in }\end{array}$ & $\begin{array}{l}\text { SH (LIFE), SI } \\
\text { (SIQ-Jr) }\end{array}$ & $2 \%$ \\
\hline
\end{tabular}




\begin{tabular}{|c|c|c|c|c|c|c|c|c|c|c|c|}
\hline & & & & & & & & & $\begin{array}{l}\text { pharmacotherapy; } 1+\text { parent/ } \\
\text { guardian willing to participate in } \\
\text { family sessions }\end{array}$ & & \\
\hline $\begin{array}{l}\text { James, Taylor, } \\
\text { Winmill, and } \\
\text { Alfoadari (2008) }\end{array}$ & Pre-post & 16 & UK & - & $100 \%$ & $15-18(16.4)$ & 12 & Outpatient & $\begin{array}{l}\text { History of }>6 \text { months severe and } \\
\text { persistent self-injury }\end{array}$ & $\begin{array}{l}\text { SH (clinical } \\
\text { interview), BPD } \\
\text { (SCID-II) }\end{array}$ & $13 \%$ \\
\hline $\begin{array}{l}\text { James, Winmill, } \\
\text { Anderson, and } \\
\text { Alfoadari (2011) }\end{array}$ & Pre-post & 18 & UK & - & $88 \%$ & $13-17(15.5)$ & 3 & Outpatient & $\begin{array}{l}\text { History of }>6 \text { months persistent } \\
\text { self-injury }\end{array}$ & $\begin{array}{l}\text { SH (clinical } \\
\text { interview), BPD } \\
\text { (SCID-II) }\end{array}$ & $28 \%$ \\
\hline Katz et al. (2004) & СCT & 53 & Canada & Treatment-as-usual & $84 \%$ & $14-17(15.4)$ & 0.5 & Inpatient & $\begin{array}{l}\text { Admitted to inpatient unit } \\
\text { following a suicide attempt or } \\
\text { with severe suicidal ideation; } \\
\text { agreed to stay in the hospital for } \\
\text { brief treatment }\end{array}$ & $\begin{array}{l}\text { SH (LPC), SI } \\
\text { (SIQ-Jr) }\end{array}$ & $9 \%$ \\
\hline McCauley et al. (2018) & RCT & 173 & USA & $\begin{array}{l}\text { Individual and } \\
\text { group } \\
\text { supportive therapy }\end{array}$ & $94 \%$ & $12-18(14.9)$ & 6 & $\begin{array}{l}\text { ED, inpatient, } \\
\text { outpatient } \\
\text { services, and } \\
\text { community } \\
\text { services }\end{array}$ & $\begin{array}{l}\text { 1+ lifetime suicide attempt; } \\
\text { elevated past-month suicidal } \\
\text { ideation ( } \geqslant 24 \text { on the SIQ-Jr); } \geqslant 3 \\
\text { lifetime self-injury episodes, } \\
\text { including } 1 \text { episode in the } 12 \\
\text { weeks before screening, } 3+\text { BPD } \\
\text { criteria }\end{array}$ & $\begin{array}{l}\text { SH (SASII), SI } \\
\text { (SIQ-Jr), BPD } \\
\text { (SCID-II) }\end{array}$ & $40 \%$ \\
\hline McDonell et al. (2010) & CCT & 155 & USA & Historical control & $58 \%$ & $12-17(15.54)$ & 12 & Inpatient & Not stated & $\begin{array}{l}\text { SH (quality } \\
\text { assurance } \\
\text { database) }\end{array}$ & $0 \%$ \\
\hline Mehlum et al. (2014) & RCT & 77 & Norway & $\begin{array}{l}\text { Enhanced usual } \\
\text { care }\end{array}$ & $88 \%$ & $\begin{array}{l}\text { 12-18 (DBT: } \\
\text { 15.9, Control: } \\
\text { 15.3) }\end{array}$ & 4.75 & Outpatient & $\begin{array}{l}2+\text { self-injury episodes, with } 1+ \\
\text { within the last } 16 \text { weeks; } 2+\text { BPD } \\
\text { criteria (and the self-destructive } \\
\text { criterion), or, } 1+\text { criterion of BPD } \\
\text { and at least } 2 \text { subthreshold-level } \\
\text { criteria; fluency in Norwegian }\end{array}$ & $\begin{array}{l}\text { SH (frequency } \\
\text { count), SI (SIQ-Jr), } \\
\text { BPD (BSL-23) }\end{array}$ & $0 \%$ \\
\hline $\begin{array}{l}\text { Perepletchikova et al. } \\
\text { (2011) }\end{array}$ & Pre-post & 11 & USA & - & $55 \%$ & $\begin{array}{l}8-11 \text { years } 6 \\
\text { months }(9.83)\end{array}$ & 1.5 & $\begin{array}{l}\text { Elementary } \\
\text { school }\end{array}$ & Not stated & SI (MFQ) & $0 \%$ \\
\hline $\begin{array}{l}\text { Rathus and Miller } \\
\text { (2002) }\end{array}$ & CCT & 13 & USA & Treatment-as-usual & $93 \%$ & $\begin{array}{l}\text { Range not } \\
\text { stated (DBT: } \\
\text { 16.1, Control: } \\
\text { 15.0) }\end{array}$ & 3 & Outpatient & $\begin{array}{l}\text { A suicide attempt with last } 16 \\
\text { weeks or current suicidal } \\
\text { ideation; a BPD diagnosis or } 3+ \\
\text { BPD criteria }\end{array}$ & SI (SSI), BPD (LPI) & $\begin{array}{l}\text { Defers by } \\
\text { group TAU: } \\
60 \% \text {, } \\
\text { DBT-A: } \\
38 \%\end{array}$ \\
\hline $\begin{array}{l}\text { Santamarina-Perez } \\
\text { et al. (2020) }\end{array}$ & RCT & 35 & Spain & $\begin{array}{l}\text { Treatment-as-usual } \\
+ \text { group therapy }\end{array}$ & $89 \%$ & $\begin{array}{l}12-17 \text { years } 11 \\
\text { months (DBT: } \\
\text { 15.3, Control: } \\
15.2 \text { ) }\end{array}$ & 4 & Outpatient & $\begin{array}{l}\text { Repetitive SH (proposed DSM-V } \\
\text { criteria) and/or suicide attempts } \\
\text { over the last } 12 \text { months and at } \\
\text { current high risk of suicide; } 1+ \\
\text { parent/guardian willing to } \\
\text { participate in family sessions }\end{array}$ & $\begin{array}{l}\text { SH (medical } \\
\text { records), SI (SIQ-Jr) }\end{array}$ & $20 \%$ \\
\hline $\begin{array}{l}\text { Tebbett-Mock et al. } \\
(2020)\end{array}$ & CCT & 801 & USA & Historical control & $66 \%$ & $\begin{array}{l}12-17 \text { (DBT): } \\
15.7 \text { (1.4) } \\
\text { Control: } 15.6 \\
\text { (1.5) }\end{array}$ & 0.36 & Inpatient & Not stated & $\begin{array}{l}\text { SH (medical } \\
\text { records), SI } \\
\text { (observation hours } \\
\text { for SI) }\end{array}$ & $\begin{array}{l}\text { Not } \\
\text { reported }\end{array}$ \\
\hline
\end{tabular}




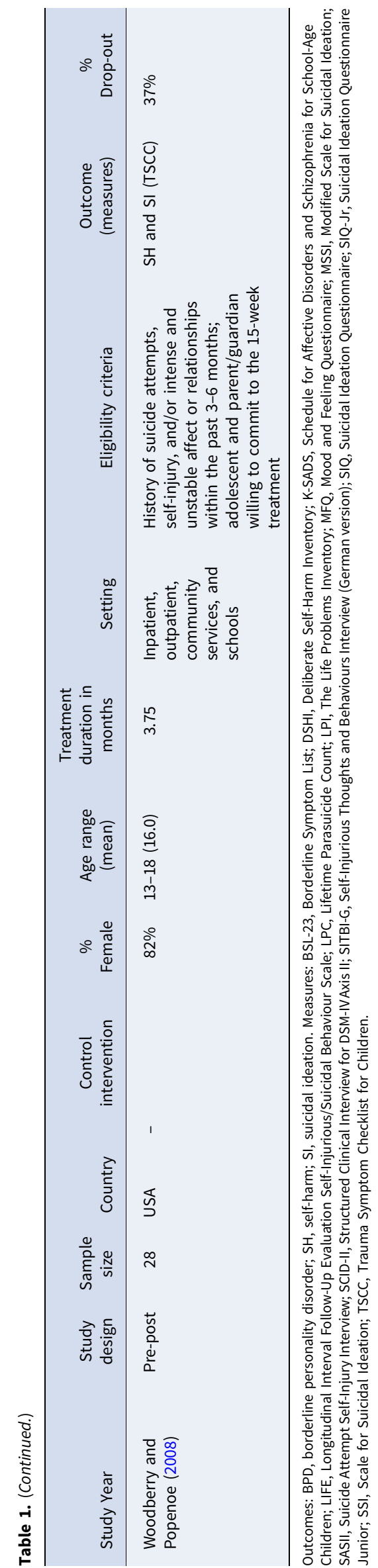

First, we considered the overall efficacy of DBT-A, compared to control interventions, for reducing adolescent self-harm. Seven effect sizes encompassing 1314 participants (DBT-A: $n=$ 714, Control: $n=600, k=7$ ) were extracted. Meta-analysis revealed a significant difference between groups $(g=-0.44,95 \%$ $\mathrm{CI}-0.81$ to $-0.07, p=0.021)$ with a high heterogeneity between studies $\left(I^{2}=80.13 \%\right)$. That is, DBT-A interventions showed a small-to-medium improvement in reducing self-harm compared to control interventions. See Fig. 2 for a comparison of DBT-A (relative to control interventions) by study type (RCT or CCT) for reducing self-harm.

Next, we considered the overall efficacy of DBT-A, compared to control interventions, for reducing adolescent suicidal ideation. Five effect sizes encompassing 1159 participants (DBT-A: $n=604$, Control: $n=555, k=6$ ) were extracted. Meta-analysis revealed a significant difference between groups $(g=-0.31,95 \%$ CI -0.52 to $-0.09, p=0.006$ ), with moderate heterogeneity between studies $\left(I^{2}=44.05 \%\right)$. That is, DBT-A was moderately more effective at reducing suicidal ideation than control interventions. See Fig. 3 for a comparison of DBT-A (relative to control interventions) by study type (RCT or CCT) for reducing suicidal ideation.

In terms of BPD symptoms, Mehlum et al. (2014) conducted the only RCT which assessed the efficacy of DBT-A in reducing BPD symptoms. They reported that both DBT-A $(n=39)$ and 'enhanced usual care' (non-manualized standard care provided at least once weekly for the purpose of the trial; $n=38$ ) reduced BPD symptoms, but found no significant group difference. Given that only one RCT was eligible for inclusion in this review, we did not conduct meta-analysis of the effect of DBT-A and control interventions on BPD symptoms.

Moving beyond group comparisons of treatment and control interventions, we next considered within-subject changes in selfharm, suicidal ideation, and BPD symptoms following DBT-A intervention. Table 2 shows the effects of DBT-A across outcome measures. Among participants who received DBT-A, pre-post comparisons indicate large reductions in self-harm $(g=-0.98)$, suicidal ideation $(g=-1.16)$, and BPD symptoms $(g=-0.97)$. All effects were statistically significant, with suicidal ideation $\left(I^{2}=54.58\right)$ and BPD symptoms $\left(I^{2}=43.51\right)$ showing moderate heterogeneity, and self-harm showing low-to-moderate heterogeneity $\left(I^{2}=0.00\right)$ across studies.

Next, in order to better understand the parameters in which DBT-A is most effective, we assessed whether study characteristics moderated the efficacy of DBT-A in reducing adolescent self-harm and suicidal ideation. We conducted multiple metaregression to assess whether treatment duration (in months), age (in years), or proportion of young women in the overall sample (compared to young men) moderated the size of meta-analytic effects between DBT-A and control interventions (see Supplement 2 for all coefficients). Treatment duration was negatively associated with the change in effect sizes for suicidal ideation $(b=-0.08, p=0.012)$, but not self-harm $(b=-0.06$, $p=0.379)$. That is, a longer duration of DBT-A led to a larger reduction in suicidal ideation when compared to control interventions. In contrast, gender composition and age of the sample did not influence the effect size of the difference between DBT-A and control interventions for self-harm or suicide ideation (all $p s>0.05)$.

We repeated these meta-regressions for the assessments of effect sizes in pre-post evaluations (see Supplement 2 for all coefficients). Again, treatment duration was negatively associated with larger effect sizes for reducing BPD symptoms $(b=-0.29$, 


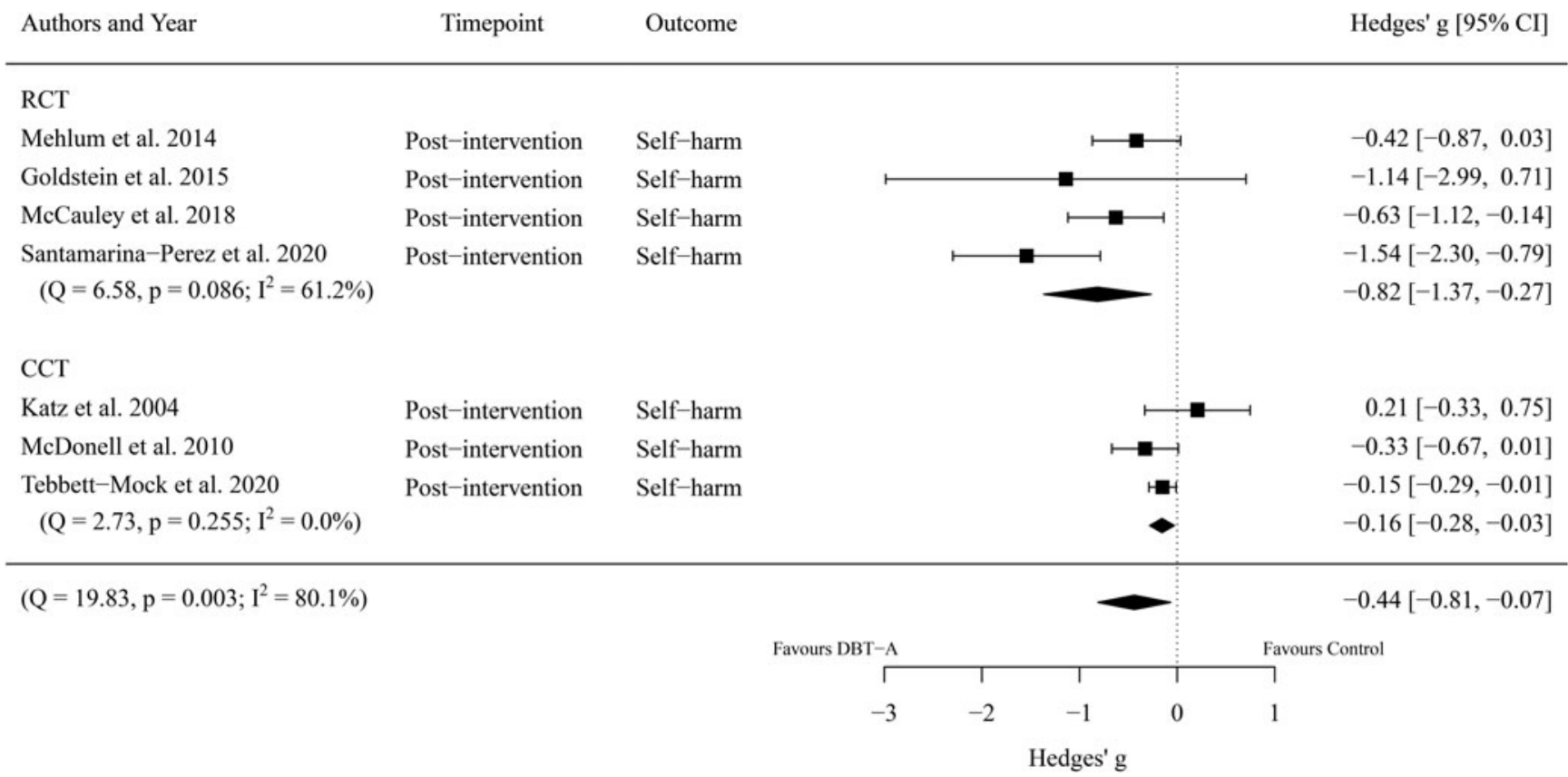

Fig. 2. Forest plot of trials comparing the effect of DBT-A and controls on symptoms of self-harm.

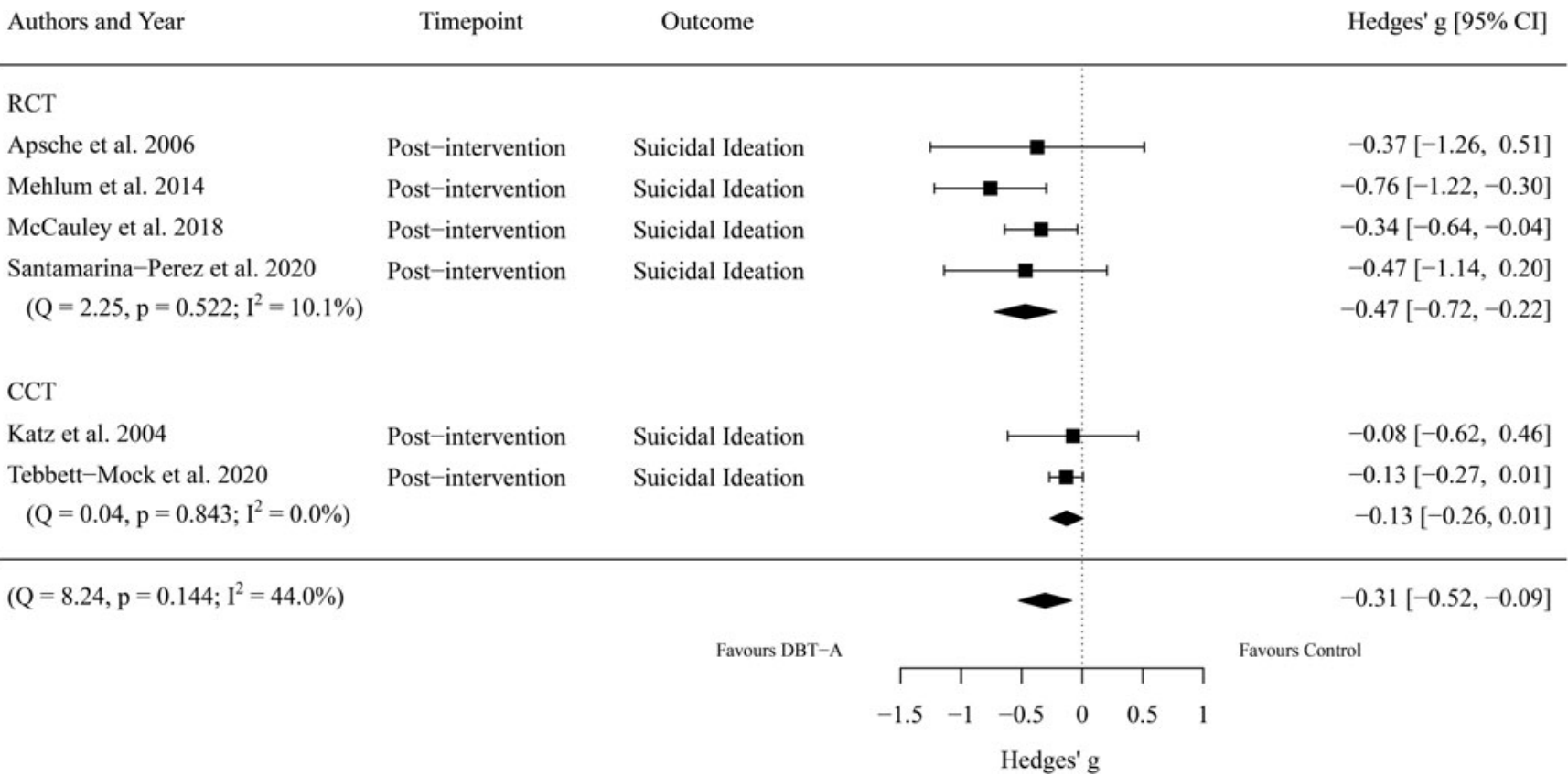

Fig. 3. Forest plot of trials comparing the effect of DBT-A and controls on symptoms of suicidal ideation.

$p=0.016$ ), such that the longer the DBT-A treatment the larger the reduction in symptoms from pre to post. No other moderating effects of treatment duration were found. Similarly, gender composition and age of the sample did not influence any of the outcomes in pre-post analyses.

We next consider the potential impact of publication bias in the studies examined in these meta-analyses. For each outcome (both in the effect as compared to control interventions and in pre-post comparisons), visual inspection of funnel plots suggested that across all four outcomes, studies were symmetrically distributed. That is, we found no evidence for publication bias in the studies assessing self-harm, suicidal ideation, or BPD symptoms included in the present meta-analyses. In addition, Egger's regression found no evidence for funnel plot asymmetry in the analyses we conducted (all $z s<1.64$, all $p s>0.100$ ). Given that no indication of publication bias was found, no adjustments according to trim-and-fill analysis were conducted in any of the analyses.

Finally, we evaluate the quality of the studies included in the current review using predefined criteria based on the Agency 
Table 2. Pre-post treatment effects (Hedges' $g$ ) and heterogeneity indices of DBT-A

\begin{tabular}{|c|c|c|c|c|c|}
\hline Outcome & $k$ & $n$ & Hedges' $g$ & $95 \% \mathrm{Cl}$ & $I^{2}$ \\
\hline \multicolumn{6}{|l|}{ Reduction at treatment completion } \\
\hline Self-harm & 16 & 498 & $-0.98^{\star}$ & -1.15 to -0.81 & 0.00 \\
\hline Suicidal ideation & 11 & 299 & $-1.16^{\star}$ & -1.51 to -0.80 & 54.58 \\
\hline Borderline personality disorder symptoms & 5 & 218 & $-0.97^{\star}$ & -1.31 to -0.63 & 43.51 \\
\hline
\end{tabular}

Hedges' $g$ indicates change from pre to post-intervention such that a negative effect size indicates a reduction in that outcome following DBT-A.

*Indicates effect sizes that are statistically significant $(p<0.001)$.

for Healthcare Research and Quality method guide (Viswanathan et al., 2018). Overall, statistical problems were common with $57.1 \%$ of studies rated at 'High' risk of bias due to low sample sizes. High risk of measurement bias (38.1\%) and confounding bias $(33.3 \%)$ was also common, due to reliance on unvalidated instruments or inadequately addressing potential confounds. Taken together, quality assessments highlight the need for widespread adoption of standardized measurement instruments and well-powered replication studies (see Supplement 1).

\section{Discussion}

Given the scarcity of studies investigating the efficacy of DBT-A in reducing adolescent self-harm and suicidal ideation, particularly in the context of comorbid psychopathology such as BPD, we conducted a systematic review of controlled trials to inform bestpractice clinical decision making. Our meta-analysis included 21 studies comprised of 1673 participants and provides evidence to support the efficacy of DBT-A (compared to control interventions) for reducing self-harm and suicidal ideation as primary outcomes. The effect size for self-harm reduction in favor of DBT-A was large for RCTs and small-to-medium when CCTs were included. The effect sizes for suicidal ideation reduction in favor of DBT-A were small-to-medium for both RCTs and when all controlled studies were included in the analysis. The current review identified an insufficient number of studies to evaluate the efficacy of DBT-A in BPD symptoms.

A growing body of research demonstrates that therapeutic interventions for self-harm and suicidal ideation in general show limited efficacy (Fox et al., 2020; Kothgassner et al., 2020), highlighting the importance of isolating specific therapies which show promise for further development. In a review of all published RCTs targeted at reducing suicidal thoughts and behaviors, DBT showed a small treatment effect for self-harm, but had no effect on suicidal ideation (Fox et al., 2020). Similarly, in a review of controlled trials investigating the efficacy of DBT among adult samples found a small effect in favor of DBT for reducing selfinjury, but no effect on suicidal ideation (DeCou, Comtois, \& Landes, 2019). Focusing specifically on children and adolescents, our results reveal promising effects of DBT-A for both self-harm and suicidal ideation for both RCTs and CCTs. Given that adolescence is a key developmental period for both self-harm and suicidal ideation (Wyman, 2014), future research is needed to understand the underlying mechanism(s) of how DBT-A works to improve self-harm and suicidal ideation.

Across all studies, our findings indicate that longer duration of DBT-A may be crucial for greater efficacy, particularly for suicidal ideation. Additionally, longer treatment duration was associated with greater reductions in BPD symptoms in pre-post evaluations. These findings correspond to the DBT-A treatment hierarchy in which further BPD symptoms are addressed in later stages of therapy, after an initial focus on establishing sufficient behavior control. Since the therapeutic relationship can be considered as a critical reinforcement for people with BPD (Bedics, Atkins, Harned, \& Linehan, 2015), a longer duration may mean a more effective use of the therapeutic relationship in terms of contingency management (Miller et al., 2017).

We also found larger effect sizes for self-harm and suicidal ideation in RCTs than in CCTs. This difference might be explained by the fact that these study types differ by recruitment setting. RCTs predominantly recruited adolescents receiving outpatient care (Goldstein et al., Mehlum et al., 2014; Santamarina-Perez et al., 2020), except for McCauley et al. (2018) who included adolescents recruited from both inpatient and outpatient settings, whereas all CCTs reporting self-harm and suicidal ideation outcomes consisted of participants recruited from inpatient settings (Katz, Cox, Gunasekara, \& Miller, 2004; McDonell et al., 2010; Tebbett-Mock, Saito, McGee, Woloszyn, \& Venuti, 2020). Another potential explanation may lie in the different methodological quality of RCT and CCT studies.

No study accounted for the combined effect of pharmacological treatment with DBT-A, despite the fact that psychopharmacological treatment for adolescents with BPD in general is common (Cailhol et al., 2013), and over half of participants treated in the included trials received additional psychopharmacological treatment. Further, some studies reported reduction of medication or adherence as an outcome variable (Katz et al., 2004; McDonell et al., 2010; Tebbett-Mock et al., 2020). However, to date the efficacy of a combined therapy approach remains unclear.

Critically, DBT-A targets both the adolescent and their family. Typically, adolescents continue to live in the environment where they acquired their dysfunctional patterns and so families are integrated into therapy in order to address invalidating behaviors within the family context. In terms of contingencies, this holistic approach reinforces skills and helps to decrease maladaptive behaviors by addressing both the adolescent's and parent's behavioral and communicative repertoire. Preliminary research provides tentative support for these mechanisms of change. In a non-randomized pilot of DBT-A among a small sample of ethnic minority adolescents, adaptive coping at pretreatment predicted subsequent increased use of DBT skills at post-treatment (Yeo et al., 2020). Secondary analysis of McCauley et al. (2018) revealed that adolescents who reported higher emotion dysregulation at baseline, and whose parents reported greater psychopathology and emotion dysregulation demonstrated greater reduction in self-harm following 6-months of DBT-A treatment (Adrian et al., 2019). In a longitudinal study of adults with a recent suicide attempt who received DBT treatment, participants with higher problem-focused coping and poorer access to emotion regulation strategies were more likely to reattempt suicide over the course of 2 years (Kuehn, King, 
Linehan, \& Harned, 2020). However, future research is needed to better establish mechanisms of therapeutic change, as well as identify who stands to benefit most from DBT-A.

Our review has several limitations. First, only one RCT (Mehlum et al., 2014) assessed the impact of DBT-A (compared to control interventions) on BPD symptoms, preventing us from meta-analytically considering change in BPD symptoms as a secondary outcome. Second, we reported a moderately high heterogeneity among studies assessing the efficacy of DBT-A on self-harm. This heterogeneity might be due to the use of different control interventions, with some studies using specific psychotherapeutic control interventions (McCauley et al., 2018) and providing enhanced usual care or additional propositions (Mehlum et al., 2014; Santamarina-Perez et al., 2020). Alternatively, this heterogeneity may reflect differences in how self-harm was assessed; $58.8 \%(k$ $=7$ ) of studies used unvalidated clinical interviews, medical records, daily diary cards, or instruments developed in-house to assess selfharm. Moreover, our meta-analysis is limited by the low number of effects available to be included which contributes to the wide confidence intervals of some of the estimates. Finally, young women made up $75 \%$ or more of the sample in most studies $(81.0 \%, k=$ $17)$ included in this review. Thus, questions remain regarding the efficacy of DBT-A in reducing self-harm and suicidal ideation among young men and gender-diverse young people.

Despite these limitations, this review provides the most comprehensive analysis to date of the available evidence for the efficacy of DBT-A in reducing self-harm and suicidal ideation among adolescents. Current evidence indicates that DBT-A is superior to control interventions in reducing both self-harm and suicidal ideation among adolescents, with limited evidence of efficacy for reducing BPD symptoms. Future research should focus on improving the quality of evidence for the efficacy of DBT-A for underrepresented populations such as young men and gender diverse people, and for reducing BPD symptoms among adolescents in general. Greater investigation is also needed to understand the combined effect of DBT-A and psychopharmacological interventions, as well as to establish the feasibility and efficacy of DBT-A in teletherapeutic contexts in order to improve accessibility for young people who require specialized care for reducing their self-harm behavior and suicidal ideation.

Supplementary material. The supplementary material for this article can be found at https://doi.org/10.1017/S0033291721001355.

Author contributions. ODK, AG, KR, and MH wrote the first draft of the manuscript. ODK and KR conducted the literature search, coded the studies, and conducted the risk of bias assessment. ODK and AG prepared the statistical procedures and AG analyzed the data and made final figures. CS and PLP contributed extensively to the first draft. All authors approved the final manuscript.

Financial support. This research received no specific grant from any funding agency, commercial or not-for-profit sectors.

Conflict of interest. None of the authors declare any conflict of interest with regard to this manuscript. Paul Plener has received research funding from the German Federal Institute for Drugs and Medical Devices (BfArM), German Federal Ministry of Education and Research (BMBF), VW-Foundation, Baden-Württemberg Foundation, Lundbeck, and Servier. He received a speaker's honorarium from Shire.

\section{References}

Studies included in the meta-analysis are denoted with an asterisk.
Adrian, M., McCauley, E., Berk, M. S., Asarnow, J. R., Korslund, K., Avina, C. \& Linehan, M. M. (2019). Predictors and moderators of recurring selfharm in adolescents participating in a comparative treatment trial of psychological interventions. Journal of child psychology and psychiatry, 60 (10), 1123-1132. https://doi.org/10.1111/jcpp.13099.

American Psychiatric Association (2006). American Psychiatric Association practice guidelines for the treatment of psychiatric disorders. Washington DC: American Psychiatric Pub.

Ammerman, B. A., Jacobucci, R., Kleiman, E. M., Uyeji, L. L., \& McCloskey, M. S. (2018). The relationship between nonsuicidal self-injury age of onset and severity of self-harm. Suicide Life-Threatening Behavior, 48(1), 31-37. doi:10.1111/sltb.12330.

*Apsche, J. A., Bass, C. K., \& Siv, A. (2006). A treatment study of mode deactivation therapy in an out patient community setting. International Journal of Behavioral Consultation and Therapy, 2(2), 277-285. doi: 10.1037/ h0100782.

Bedics, J. D., Atkins, D. C., Harned, M. S., \& Linehan, M. M. (2015). The therapeutic alliance as a predictor of outcome in dialectical behavior therapy versus nonbehavioral psychotherapy by experts for borderline personality disorder. Psychotherapy, 52(1), 67. doi:10.1037/a0038457.

${ }^{*}$ Berk, M. S., Starace, N. K., Black, V. P., \& Avina, C. (2020). Implementation of dialectical behavior therapy with suicidal and self-harming adolescents in a community clinic. Archives of Suicide Research, 24(1), 64-81. doi:10.1080/ 13811118.2018.1509750.

Bertolote, J. M., \& Fleischmann, A. (2002). Suicide and psychiatric diagnosis: A worldwide perspective. World Psychiatry, 1(3), 181-185.

*Buerger, A., Fischer-Waldschmidt, G., Hammerle, F., Auer, K. V., Parzer, P., \& Kaess, M. (2019). Differential change of borderline personality disorder traits during dialectical behavior therapy for adolescents. Journal of Personality Disorders, 33(1), 119-134. doi:10.1521/pedi_2018_32_334.

Cailhol, L., Jeannot, M., Rodgers, R., Guelfi, J. D., Perez-Diaz, F., Pham-Scottez, A., ... Speranza, M. (2013). Borderline personality disorder and mental healthcare service use among adolescents. Journal of Personality Disorders, 27(2), 252-259. doi:10.1521/pedi.2013.27.2.252.

Card, N. A. (2012). Applied meta-analysis for social science research. New York, NY: Guilford.

Chapman, A. L. (2006). Dialectical behavior therapy: Current indications and unique elements. Psychiatry, 3(9), 62-68

Cochrane Collaboration (2014). Review manager (RevMan) [computer program]. version 5.3. Copenhagen: The Nordic Cochrane Centre.

Cohen, J. (1988). Statistical power analysis for the behavioral sciences. New York, NY: Routledge Academic.

Collaboration GBoDP (2016). Global and national burden of diseases and injuries among children and adolescents between 1990 and 2013: Findings from the global burden of diseases and injuries among children and adolescents between 1990 and 2013: Findings from the global burden of disease 2013 study. JAMA Pediatrics, 170(3), 267-287. doi:10.1001/ jamapediatrics.2015.4276.

${ }^{*}$ Courtney, D. B., \& Flament, M. F. (2015). Adapted dialectical behavior therapy for adolescents with self-injurious thoughts and behaviors. The Journal of Nervous and Mental Disease, 203(7), 537-544. doi: 10.1097/ NMD.0000000000000324.

Cristea, I. A., Gentili, C., Cotet, C. D., Palomba, D., Barbui, C., \& Cuijpers, P. (2017). Efficacy of psychotherapies for borderline personality disorder: A systematic review and meta-analysis. JAMA Psychiatry, 74(4), 319-328. doi:10.1001/jamapsychiatry.2016.4287

DeCou, C. R., Comtois, K. A., \& Landes, S. J. (2019). Dialectical behavior therapy is effective for the treatment of suicidal behavior: A meta-analysis. Behavior Therapy, 50(1), 60-72. doi:10.1016/j.beth.2018.03.009.

Dunlap, W. P., Cortina, J. M., Vaslow, J. B., \& Burke, M. J. (1996). Meta-analysis of experiments with matched groups or repeated measures designs. Psychological Methods, 1(2), 170-177. https://doi.org/10.1037/1082989X.1.2.170.

Duval, S., \& Tweedie, R. (2000). A nonparametric 'trim and fill' method of accounting for publication bias in meta-analysis. Journal of the American Statistical Association, 95(449), 89-98.

Ferrara, M., Terrinoni, A., \& Williams, R. (2012). Non-suicidal self-injury (Nssi) in adolescent inpatients: assessing personality features and attitude 
toward death. Child and Adolescent Psychiatry and Mental Health, 6(1), 1-8. https://doi.org/10.1186/1753-2000-6-12.

${ }^{*}$ Fischer, S., \& Peterson, C. (2015). Dialectical behavior therapy for adolescent binge eating, purging, suicidal behavior, and non-suicidal self-injury: A pilot study. Psychotherapy, 52(1), 78-92. doi:10.1037/a0036065.

${ }^{*}$ Fleischhaker, C., Böhme, R., Sixt, B., Brück, C., Schneider, C., \& Schulz, E. (2011). Dialectical behavioral therapy for adolescents (DBT-A): A clinical trial for patients with suicidal and self-injurious behavior and borderline symptoms with a one-year follow-up. Child and Adolescent Psychiatry and Mental Health, 5(1), 3. doi:10.1186/1753-2000-5-3.

Fox, K. R., Huang, X., Guzmï ¿1/2n, E. M., Funsch, K. M., Cha, C. B., Ribeiro, J. D., \& Franklin, J. C. (2020). Interventions for suicide and self-injury: A meta-analysis of randomized controlled trials across nearly 50 years of research. Psychological Bulletin, 146(12), 1117-1145. https://doi.org/ 10.1037/bul0000305.

${ }^{*}$ Geddes, K., Dziurawiec, S., \& Lee, C. W. (2013). Dialectical behaviour therapy for the treatment of emotion dysregulation and trauma symptoms in selfinjurious and suicidal adolescent females: A pilot programme within a community-based child and adolescent mental health service. Psychiatry Journal, 2013, 145219. doi:10.1155/2013/145219.

${ }^{*}$ Gillespie, C., Joyce, M., Flynn, D., \& Corcoran, P. (2019). Dialectical behaviour therapy for adolescents: A comparison of 16-week and 24-week programmes delivered in a public community setting. Child and Adolescent Mental Health, 24(3), 266-273. doi:10.1111/camh.12325.

Gillies, D., Christou, M. A., Dixon, A. C., Featherston, O. J., Rapti, I., Garcia-Anguita, A., ... Christou, P. A. (2018). Prevalence and characteristics of self-harm in adolescents: Meta-analyses of community-based studies 1990-2015. Journal of the American Academy of Child \& Adolescent Psychiatry, 57(10), 733-741. doi:10.1016/j.jaac.2018.06.018.

Glenn, C. R., Franklin, J. C., \& Nock, M. K. (2015). Evidence-based psychosocial treatments for self-injurious thoughts and behaviors in youth. Journal of Clinical Child \& Adolescent Psychology, 44(1), 1-29. doi:10.1080/15374416.2014.945211

Goldstein, T. R., Axelson, D. A., Birmaher, B., \& Brent, D. A. (2007). Dialectical behavior therapy for adolescents with bipolar disorder: A 1-year open trial. Journal of the American Academy of Child \& Adolescent Psychiatry, 46(7), 820-830. doi:10.1097/chi.0b013e31805c1613.

${ }^{*}$ Goldstein, T. R., Fersch-Podrat, R. K., Rivera, M., Axelson, D. A., Merranko, J., Yu, H., ... Birmaher, B. (2015). Dialectical behavior therapy for adolescents with bipolar disorder: Results from a pilot randomized trial. Journal of Child and Adolescent Psychopharmacology, 25(2), 140-149. doi:10.1089/ cap.2013.0145.

Groschwitz, R. C., Plener, P. L., Kaess, M., Schumacher, T., Stoehr, R., \& Boege, I. (2015). The situation of former adolescent self-injurers as young adults: A follow-up study. BMC Psychiatry, 15(1), 1-9. doi:10.1186/s12888015-0555-1.

Hamza, C. A., Stewart, S. L., \& Willoughby, T. (2012). Examining the link between nonsuicidal self-injury and suicidal behavior: A review of the literature and an integrated model. Clinical Psychology Review, 32(6), 482-495. doi:10.1016/j.cpr.2012.05.003.

Hawton, K., Saunders, K. E., \& O'Connor, R. C. (2012). Self-harm and suicide in adolescents. The Lancet, 379(9834), 2373-2382. doi:10.1016/S0140-6736 (12)60322-5.

Higgins, J. P., Thompson, S. G., Deeks, J. J., \& Altman, D. G. (2003). Measuring inconsistency in meta-analyses. BMJ, 327(7414), 557-560. doi:10.1136/bmj.327.7414.557.

${ }^{\star} J a m e s$, A. C., Taylor, A., Winmill, L., \& Alfoadari, K. (2008). A preliminary community study of dialectical behaviour therapy (DBT) with adolescent females demonstrating persistent, deliberate self-harm (DSH). Child and Adolescent Mental Health, 13(3), 148-152. doi:10.1111/j.1475-3588.2007. 00470.x.

${ }^{\star} J a m e s$, A. C., Winmill, L., Anderson, C., \& Alfoadari, K. (2011). A preliminary study of an extension of a community dialectic behaviour therapy (DBT) programme to adolescents in the looked after care system. Child and Adolescent Mental Health, 16(1), 9-13. doi:10.1111/j.1475-3588.2010. 00571.x.

Kaplan, C., Tarlow, N., Stewart, J. G., Aguirre, B., Galen, G., \& Auerbach, R. P. (2016). Borderline personality disorder in youth: The prospective impact of child abuse on non-suicidal self-injury and suicidality. Comprehensive Psychiatry, 7, 86-94. doi:10.1016/j.comppsych.2016.08.016.

${ }^{\star}$ Katz, L. Y., Cox, B. J., Gunasekara, S., \& Miller, A. L. (2004). Feasibility of dialectical behavior therapy for suicidal adolescent inpatients. Journal of the American Academy of Child \& Adolescent Psychiatry, 43(3), 276-282. doi:10.1097/00004583-200403000-00008.

Kothgassner, O. D., Robinson, K., Goreis, A., Ougrin, D., \& Plener, P. L. (2020). Does treatment method matter? A meta-analysis of the past 20 years of research on therapeutic interventions for self-harm and suicidal ideation in adolescents. Borderline Personality Disorder and Emotion Dysregulation, 7, 9. doi:10.1186/s40479-020-00123-9.

Kuehn, K. S., King, K. M., Linehan, M. M., \& Harned, M. S. (2020). Modeling the suicidal behavior cycle: Understanding repeated suicide attempts among individuals with borderline personality disorder and a history of attempting suicide. Journal of Consulting and Clinical Psychology, 88, 570-581. doi: $10.1037 /$ ccp0000496.

Lakens, D. (2013). Calculating and reporting effect sizes to facilitate cumulative science: A practical primer for $t$ tests and ANOVAs. Frontiers in Psychology, 4, 863. doi: $10.3389 /$ fpsyg. 2013.00863

Linehan, M. M., Heard, H. L., \& Armstrong, H. E. (1993). Naturalistic follow-up of a behavioral treatment for chronically parasuicidal borderline patients. Archives of General Psychiatry, 50(12), 971-974. doi: 10.1001/ archpsyc. 1993.01820240055007

Lipsey, M. W., \& Wilson, D. B. (2001). Practical meta-analysis. Thousand Oaks, CA: Sage.

${ }^{*}$ McCauley, E., Berk, M. S., Asarnow, J. R., Adrian, M., Cohen, J., Korslund, K., ... Linehan, M. M. (2018). Efficacy of dialectical behavior therapy for adolescents at high risk for suicide: A randomized clinical trial. JAMA Psychiatry, 75(8), 777-785. doi: 10.1001/jamapsychiatry.2018.1109.

${ }^{*}$ McDonell, M. G., Tarantino, J., Dubose, A. P., Matestic, P., Steinmetz, K., Galbreath, H., \& McClellan, J. M. (2010). A pilot evaluation of dialectical behavioural therapy in adolescent long-term inpatient care. Child and Adolescent Mental Health, 15(4), 193-196. doi:10.1111/j.1475-3588.2010. 00569.x.

${ }^{*}$ Mehlum, L., Tørmoen, A. J., Ramberg, M., Haga, E., Diep, L. M., Laberg, S., ... Grøholt, B. (2014). Dialectical behavior therapy for adolescents with repeated suicidal and self-harming behavior: A randomized trial. Journal of the American Academy of Child \& Adolescent Psychiatry, 53(10), 1082-1091. doi:10.1016/j.jaac.2014.07.003.

Miller, A. L., Rathus, J. H., \& Linehan, M. M. (2017). Dialectical behavior therapy with suicidal adolescents. New York: Guilford Press.

National Collaborating Center for Mental Health (2009). Borderline personality disorder: The NICE guideline on treatment and management. Leicester/ London: The British Psychological Society \& The Royal College of Psychiatrists; [updated July 2018. Available from: https://www.nice.org.uk/ guidance/cg78.].

Nock, M. K., Joiner Jr T. E., Gordon, K. H., Lloyd-Richardson, E., \& Prinstein, M. J. (2006). Non-suicidal self-injury among adolescents: Diagnostic correlates and relation to suicide attempts. Psychiatry Research, 144(1), 65-72. doi:10.1016/j.psychres.2006.05.010.

${ }^{\star}$ Perepletchikova, F., Axelrod, S. R., Kaufman, J., Rounsaville, B. J., Douglas-Palumberi, H., \& Miller, A. L. (2011). Adapting dialectical behaviour therapy for children: Towards a new research agenda for paediatric suicidal and non-suicidal self-injurious behaviours. Child and Adolescent Mental Health, 16(2), 116-121. doi:10.1111/j.1475-3588.2010. 00583.x.

Pigott, T. D. (2012). Advances in meta-analysis. New York, NY: Springer.

Plener, P. L., Schumacher, T. S., Munz, L. M., \& Groschwitz, R. C. (2015). The longitudinal course of non-suicidal self-injury and deliberate self-harm: A systematic review of the literature. Borderline Personal Disorder and Emotion Dysregulation, 2, 2. doi:10.1186/s40479-014-0024-3.

${ }^{*}$ Rathus, J. H., \& Miller, A. L. (2002). Dialectical behavior therapy adapted for suicidal adolescents. Suicide and Life-Threatening Behavior, 32(2), 146-157.

Rathus, J. H., \& Miller, A. L. (2015). DBT skills manual for adolescents. New York: Guilford Publications.

Ribeiro, J. D., Franklin, J. C., Fox, K. R., Bentley, K. H., Kleiman, E. M., Chang, B. P., \& Nock, M. K. (2016). Self-injurious thoughts and behaviors as risk factors for future suicide ideation, attempts, and death: A meta-analysis 
of longitudinal studies. Psychological Medicine, 46(2), 225-236. doi:10.1017/ S0033291715001804

*Santamarina-Perez, P., Mendez, I., Singh, M. K., Berk, M., Picado, M., Font, E., ... Cosi, A. (2020). Adapted dialectical behavior therapy for adolescents with a high risk of suicide in a community clinic: A pragmatic randomized controlled trial. Suicide and Life-Threatening Behavior, 50(3), 652-667. 10.1111/sltb.12612.

Sterne, J. A., \& Egger, M. (2005). Regression methods to detect publication and other bias in meta-analysis. Publication bias in Meta-analysis: Prevention, Assessment and Adjustments, 54(10), 99-110. doi:10.1002/0470870168.ch6.

${ }^{\star}$ Tebbett-Mock, A. A., Saito, E., McGee, M., Woloszyn, P., \& Venuti, M. (2020). Efficacy of dialectical behavior therapy versus treatment as usual for acute-care inpatient adolescents. Journal of the American Academy of Child \& Adolescent Psychiatry, 59(1), 149-156. doi:10.1016/j.jaac.2019.01.020.

Viechtbauer, W. (2010). Conducting meta-analyses in R with the metafor package. Journal of Statistical Software, 36(3), 1-48. doi:10.18637/ jss.v036.i03
Viswanathan, M., Patnode, C. D., Berkman, N. D., Bass, E. B., Chang, S., Hartling, L., ... Kane, R. L. (2018). Recommendations for assessing the risk of bias in systematic reviews of health-care interventions. Journal of Clinical Epidemiology, 97, 26-34. doi: 10.1016/j.jclinepi.2017. 12.004 .

*Woodberry, K. A., \& Popenoe, E. J. (2008). Implementing dialectical behavior therapy with adolescents and their families in a community outpatient clinic. Cognitive and Behavioral Practice, 15(3), 277-286. doi:10.1016/ j.cbpra.2007.08.004.

Wyman, P. A. (2014). Developmental approach to prevent adolescent suicides: Research pathways to effective upstream preventive interventions. American Journal of Preventive Medicine, 47(3), S251-S256. doi:10.1016/ j.amepre.2014.05.039.

Yeo, A. J., Germán, M., Wheeler, L. A., Camacho, K., Hirsch, E., \& Miller, A. (2020). Self-harm and self-regulation in urban ethnic minority youth: A pilot application of dialectical behavior therapy for adolescents. Child and Adolescent Mental Health, 25(3), 127-134. 10.1111/camh.12374. 